\title{
Opto-Electronic Oscillator with Mach-Zender Modulator
}

\author{
Alexander A. Bortsov and Sergey M. Smolskiy, Member IEEE
}

\begin{abstract}
The non-standard opto-electronic oscillator (OEO) operation is discussed in the generation mode of a single-side optical harmonic on the base of external and internal modulation of the laser signal. The OEO mathematical model is formed basing on laser differential equations for the closed radiofrequency network. With the help of the model offered, the phase noise of OEO radiofrequency oscillations is analyzed. It is shown that phase noise reduction in the circuit with external modulation depends not only on the increased laser power and growth of the geometric length of the optical fiber, but on reduction of the laser phase noise.
\end{abstract}

Keywords: opto-electronic oscillator, phase noise, optical fiber, QW laser, microwave oscillator.

\section{INTRODUCTION. THE OPTO-ELECTRONIC OSCILLATOR STRUCTURE}

Development and creation of the compact ultra-low-noise microwave signal sources, which would be impact-resistant, is an important problem of modern radio-physics and radio engineering. Levels of the phase noise spectral density at the microwave source output must be for most of the applications $-120 \ldots-170 \mathrm{~dB} / \mathrm{Hz}$ at generation frequency $8 \ldots 12 \mathrm{GHz}$ for $1-$ $\mathrm{kHz}$ offset from a carrier. Constructions of these oscillators must sustain the strong mechanical impact loads in $200 \ldots 2000 \mathrm{~N} / \mathrm{cm}$ and high accelerations up to $2 \ldots 10 \mathrm{~g}$. Geometrical dimensions of the modern signal sources should often be approximately 10x10x10 cubic mm, especially for the satellite applications.

Development and implementation of new compact microwave and millimeter-wave oscillators with improved performance would lead to revolutionary jump in radio electronics, perhaps, comparable to discovery of the quantumdimensional lasers or (as in radio engineering) at arriving of the high-stability quartz crystal resonator. The new type of oscillators called as opto-electronic oscillator (OEO) described in this paper will permit to use in the mobile communications and in Internet systems of new radiofrequency channels for information transmission, including $30 \ldots 75-\mathrm{GHz}$ ranges at the low power of transmitters. A number of publications devoted to OEO experimental investigations grows each year [1-8].

Opto-electronic oscillators will undoubtedly find wide application in the fiber-optical communication lines as well as in on-board radar systems on millimeter- and centimeter

${ }^{1}$ Alexander A. Bortsov is with Moscow Power Engineering Institute (National Research University), Russia (e-mail: 1aseroeo5@gmail.com).

Sergey M. Smolskiy is with Moscow Power Engineering Institute (National Research University), Russia (e-mail: SmolskiySM@mail.ru). ranges, in communication systems as low-noise local oscillators in receivers and as a master clock in transmitters, in an optical lidar technology, as sensors of different physical quantities and in many other systems [8-11].

Let us consider the OEO structural diagram with external modulation of optical emission, which is often called as an opto-electronic oscillator with the Max-Zender modulator (OEO MZ) presented in Figure 1a.

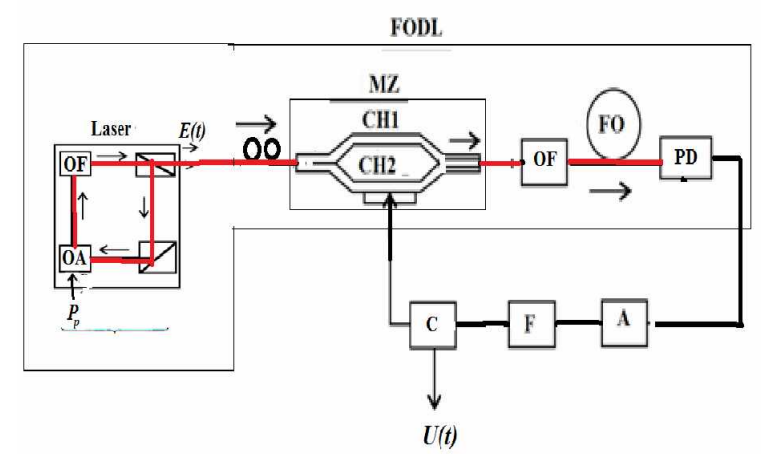

(a)

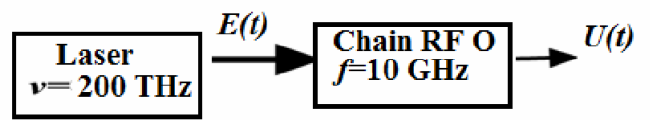

(b)

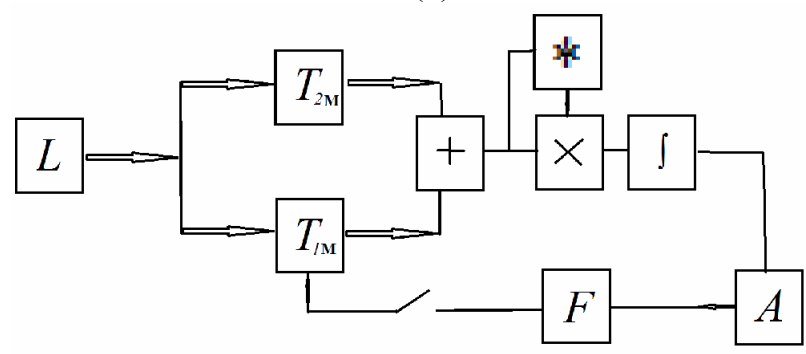

(c)

Figure 1. (a) Structural diagram of OEO with external MZ modulator; (b) OEO circuit, a laser is the energy pump; (c) Equivalent $\mathrm{OEO}$ circuit with a correlator.

OEO is formed by the following principal units: a laser, the Mach-Zender (MZ) modulator, which is connected serially into a ring, the fiber-optical system (FOS) containing an optical filter (OF) and the single-mode optical fiber (FO), a photo-detector (PD), for instance, the quantum-dimension photo-diode, a narrowband radiofrequency filter $(\mathrm{F})$, a 
nonlinear amplifier (A), and a directional coupler (C). Serially connected Laser, MZ, OF, FO, and PD constitute the unit of fiber-optical delay line (FODL), which electrical input is the $\mathrm{MZ}$ modulator input (the MZ input signal), and its electrical output coincides with PD electrical output i.e., the output voltage on the PD load resistor).

As we see, OEO contains the feedback loop of radiofrequency and it results in double generation: laser on the optical frequency and RF feedback circuit with RF modulation of the optical oscillation.

The opto-electronic oscillator implemented according to the scheme of external modulation can be considered as an oscillator with delayed feedback in which FODL of the radiofrequency oscillation is active. The FODL has its own amplitude and phase noises which are the laser noises reduced to the FODL output (or passed from the Laser output through $\mathrm{MZ}, \mathrm{OF}, \mathrm{FO})$.

We can use the quantum-dimension laser diode or the fiber-optical laser with activated erbium dope etc. as the OEO laser. In the diagram in Figure 1, the Laser is presented by closed into a loop the optical amplifier (OA), the optical filter $(\mathrm{OF})$, which corresponds to the "traveling-wave" laser or the fiber-optical laser. The optical pump power $P_{P}$ acts at the active amplifier. If the excitation conditions are met, the laser generates optical oscillations which pass from its output into $\mathrm{MZ}$, then pass via two optical channels with different delays, combine together and through OF and FO acts to the lightsensitive $\mathrm{PD}$ area. An effective modulation by $\mathrm{MZ}$ is possible in microwave range only for single-mode single-frequency and linear-polarized emission of the highly-coherent laser. Quantum-Well (QW) laser diodes and the fiber-optical lasers with polarizers at their outputs are such emission sources.

The laser is the pump source for the radiofrequency network (Figure 1b) closed into a loop and formed by a modulator, an optical fiber, a photo-detector, an electronic amplifier, an electric filter, and a coupler.

As a result of oscillation processes, the spectra are formed with fluctuations having the various nature, but the spectral line width of radiofrequency oscillations is defined by parameters of two oscillating system: the laser and the radiofrequency oscillator.

\section{PROBLEM STATEMENT}

At present, in large-dimension models of laser OEO (Figure 1) with the fiber-optical delay line the low phase noise level of $-157 \mathrm{~dB} / \mathrm{Hz}[5,6]$ is achieved on the $10 \mathrm{GHz}$ generation frequency at $1 \mathrm{kHz}$ offset from a carrier.

Experimental and theoretical investigations of the power spectral density of the laser oscillator phase noise described in [13], show that reduction of the phase noise level of OEO in many respects depends on the laser phase noise level. At oscillation frequency $8 \ldots 10 \mathrm{GHz}$ at standard offsets from 1 to $10 \mathrm{kHz}$, the power spectral density of the phase noise is -120 $\mathrm{dB} / \mathrm{Hz} . . .-140 \mathrm{~dB} / \mathrm{Hz}$.

Appearance on the commercial market of nano-dimension optical fibers with low losses (down to $0.001 \mathrm{~dB}$ per one bend, at small bend radii up to $2 \ldots .5 \mathrm{~mm}$ ) becomes the stimulus for improvement of OEO radiofrequency generation methods. This allows implementation of comparably small (by geometric linear maximal dimensions) fiber-optical 5- $\mu \mathrm{s}$ delay lines of $10 \ldots 30 \mathrm{~mm}$.

In spite of the growth of publications devoted to OEO experimental investigations, the theoretical analysis and systematization of main mechanisms of the phase noise suppression in the low-noise laser OEO was not yet described in known literature. The laser phase noise influence on the OEO radiofrequency phase noise was not researched yet.

The aim of this paper is to analyze of the main mechanisms of the phase noise suppression in the ultra-low-noise laser $O E O$ and research of $O E O$ phase noise influence from the laser phase noise, the time constant of the laser resonator, the geometric length of the optical fiber, and optical power.

Following to an approach described in [13], for OEO noise analysis, we consider the system in Figure 1, in which two different oscillation processes are developed: laser oscillations with the generation frequency of approximately $200 \mathrm{THz}$ and $10-\mathrm{GHz}$ oscillations in the radiofrequency network closed into a loop. At that, the frequency multiplicity is approximately 20,000 .

\section{LASER IN OEO}

We consider that our laser is highly-coherent device, i.e., the spectral line is much less than $100 \mathrm{MHz}$ and the mean generation frequency is $200 \mathrm{THz}$. We assume that oscillations of the normalized electromagnetic field (EMF) at the laser output are close to sinusoidal with the phase noise component $\varphi_{L m}(t)$ and normalized amplitude noises $m_{L m}(t)$ :

$$
E_{L}(t)=\left[E_{0 L}+m_{L m}(t)\right] \cos \left[2 \pi v_{0 L} t+\varphi_{0 L}+\varphi_{L m}(t)\right] .
$$

Here $E_{L}(t), E_{0 L}, m_{L m}(t)$ are normalized non-dimensional quantities, respectively: the instantaneous intensity, the EMF intensity amplitude, and the EMF amplitude noise, $v_{0 L}$ is the average laser oscillation frequency, $\varphi_{0 L}$ is the initial constant phase shift, $t$ is the current time.

In the opto-electronic oscillator system, under fulfillment of excitation conditions in the electronic part of such an oscillator, the radiofrequency oscillations $u=u_{g}(t)$ give rise.

At that, the radiofrequency signal passes to the electric $\mathrm{MZ}$ input from the output of a nonlinear amplifier through the $\mathrm{C}$ coupler during oscillation generation. The instantaneous voltage of this signal is

$$
u_{g}(t)=\left[U_{10 M Z}+m_{e m}(t)\right] \cos \left[2 \pi f t+\phi_{0 e}+\varphi_{e m}(t)\right],
$$

where $U_{01 M Z}=U_{01 C}$ is the amplitude of fundamental oscillation at the electric input of the MZ modulator or at the C output, $f$ is the oscillation radiofrequency, $\phi_{0 e}$ is the constant phase shift, $\varphi_{e m}(t)$ are electronic phase fluctuations, $m_{e m}(t)$ are electronic amplitude fluctuations.

The low-noise single-mode and single-frequency quantumdimension laser diodes or the fiber optical lasers are used as the light sources in OEO.

The laser included in the OEO structure (Figure 1) is formed by (closed in the loop) the nonlinear OA, the narrowband optical filter (OF), and the optical delay line. The 
optical oscillation frequency $v_{0 L}$, which is generated by the quantum-dimension laser diodes in the autonomous steadystate, can be found (under excitation condition fulfillment) on the basis of the phase balance equations solution for the steady-state optical intensity oscillations in the optical resonator and in the laser active element.

To reveal the main mechanisms of the laser noise influence on the OEO radiofrequency noise, the laser can be described by a system of semi-classical equation with the Langevin's sources of the white noise $\left(\xi_{E}, \xi_{P}, \xi_{N}\right)$, relatively, for the EMF intensity $E_{L}$, a polarization of the laser active material $P_{n}$, a population difference $N$. We studied the laser equation system under its operation in the single-frequency single-mode regime. At that, oscillation are linear-polarized. The main assumption for utilization of semi-classical equations is that the carrier life time on the upper operation level and the time constant $T_{0 F}$ of the laser optical filter (OF) are much larger than the relaxation time of polarization $T_{2}$. At that, the equation system with the Langevin's sources for the laser can be written as:

$$
\left\{\begin{array}{l}
\frac{d^{2} E_{L}}{d t^{2}}+\frac{1}{T_{0 F}} \frac{d E_{L}}{d t}+\left(2 \pi v_{0 F}\right)^{2} E_{L}=\frac{d^{2} P_{n}}{\varepsilon_{0} d t^{2}}+\xi_{E} \\
\frac{d^{2} P_{n}}{d t^{2}}+\frac{1}{T_{2}} \frac{d P_{n}}{d t}+\left(2 \pi v_{12}\right)^{2} P_{n}=\frac{p_{e}^{2}}{h} N E_{L}+\xi_{P} \\
\frac{d N}{d t}=\alpha_{N 0} \cdot J_{0 N}-\frac{N}{T_{1}}-G_{0} \cdot P_{n} E_{L}+\xi_{N}
\end{array}\right.
$$

In (3) $T_{2}$ is the polarization time constant, the excited particles at the upper energy level, $T_{1}$ is the lifetime of the excited particles at the upper energy level, $T_{0 F}$ is the time constant of the optical resonator, $p_{e}$ is the combined dipole moment, $h$ is the Planck constant, $v_{0 F}$ is the natural frequency of the optical resonator on the specific n-th longitudinal mode, $v_{12}$ is the optical frequency of the transition, $J_{0}$ is the constant pump current, $\alpha_{N 0} \cdot J_{0}=\left(N_{02}-N_{01}\right) /\left(N_{02} T_{1}\right)$ is the constant pump, $\varepsilon_{0}$ is the electrical constant, $v_{0 F}$ is the intrinsic optical frequency of the resonator, $P_{n}$ is the polarization of the active material, $N=\left(N_{02}-N_{01}\right)$ is the population difference between the excited and unexcited levels produced by the pumping, $G_{0}$ gain factor .

It should be noted that equations (3) are similar to wellstudied equations in the oscillator theory for the double-circuit autonomous oscillator with the inertial auto-bias chain with fluctuations.

\section{MACH-ZENDER MODULATOR IN OEO: THE EMF CORRELATOR}

An effect of the $u_{g}(t)$ voltage in the one from two MZ optical channels (Figure 1) on the refraction index of the electric-optical material (the lithium niobate) from which the MZ optical channels are made, leads to phase modulation of optical oscillation. Adding of two emission on the PD area, which passed through different optical MZ channels, leads to intensity modulation of the laser emission.

Retarded output emissions of the first and second channels of the MZ modulator $E_{1 L}$ and $E_{2 L}$ pass to the input of PD. If the fiber-optical system (FOS) is formed by single OF, the delay difference is determined as $\Delta T_{\mathrm{M}}=T_{\mathrm{M} 20}-T_{\mathrm{M} 10}$.

We considered OEO with the Mach-Zender interferometer, which perform a role of optical emission modulator as a correlator of two optical oscillations $E_{L}=E_{L}(t)$ and delayed by some time $\Delta t$ oscillation $E_{L \tau}=E_{L}(t-\Delta t)$ :

$$
R_{E}(\tau)=\left\langle E_{L}(t) E_{L \tau}^{*}(t-\Delta t)\right\rangle=\frac{1}{\tau} \int_{0}^{\tau} E_{L} E_{L \tau}^{*} d t,
$$

where $\tau$ - the time of observation. To the PD area in OEO occurs at high optical power (in contrast to fiber optic systems), so the law of random distribution (amplitude $m_{L m}$ and phase $\varphi_{L m}$ of the laser oscillation) are considered normal.

Figure 1c shows an equivalent circuit of OEO. Here the following blocks are introduced: « ++$\rangle$ (adding), «X » (multiplication), $\left\langle * »\right.$ (conjugate) $« \int »$ (integration). Blocks L, A, F designate a laser, an electronic amplifier and an electronic filter, relatively. Blocks $" T_{1 M}$ " and $\left\langle T_{2 M}\right.$ » are delays in optical channels $\mathrm{MZ}$ and $\mathrm{OF}$ and equal to: $T_{1 M}=T_{M 10}+T_{B C}$ and $T_{2 M}=T_{M 20}+T_{B C}$. At open switch, the PD photo-current is $S_{P D} R_{E}(\tau)$, where $S_{P D}$ is the PD sensitivity. When this switch is open, there is a strong correlation between random quantities of $m_{L m}$ and $\varphi_{L m}$ of different optical MZ channels due to a small delay difference of optical oscillations (tens picosecond)). At closed switch in Figure 1c), the electric oscillation affecting on the second optical MZ channel is delayed with respect to the optical oscillation of the first channel by the delay time in the optical fiber (the delay $1 \ldots 20 \mu \mathrm{s}$ ). Interchannel correlation of random quantities becomes weak. Therefore, requirements to phase noise smallness are essentially increased.

The normalized correlation function of oscillation $E_{L}=E_{L}(t)$ at the $\mathrm{MZ}$ output depends on time, and it unambiguously determines the frequency spectrum of oscillation power.

We take into account that oscillations $E_{1 L}=k_{01} E_{0 L}$ and $E_{2 L}=k_{02} E_{0 L}$ are propagated through the different $\mathrm{MZ}$ optical channels, where $k_{01}$ and $k_{02}$ are excitation coefficients $\left(k_{01}+k_{02} \approx 1\right)$. We introduce the excitation irregularity 
coefficient of $\mathrm{MZ}$ optical channels $\gamma=\left(k_{02} / k_{01}\right) \approx 1$ (in the experiment $k_{01} \approx k_{02} \approx 0.5$ ), then the result on interferencing oscillations after $\mathrm{MZ}$ is $E_{12 L}$. Let us extract the modulus and the argument of $E_{12 L}$ :

$$
\begin{aligned}
& \left|E_{12 L}\right|^{2}=E_{0 L}^{2}\left\{k_{01}{ }^{2}+k_{02}{ }^{2}-2 k_{01} k_{02} \cos \left(\varphi_{0 L 1}\left(u_{g}\right)-\varphi_{0 L 2}\right)\right\} \\
& =\frac{E_{0 L}^{2}}{2} \gamma\left\{\frac{\left(1+\gamma^{2}\right)}{2 \gamma}-\cos \left(\varphi_{0 L 1}\left(u_{g}\right)-\varphi_{0 L 2}\right)\right\} \text {. } \\
& \arg E_{12 L}=\arctan \left\{\frac{k_{01} \sin \varphi_{1}+k_{02} \sin \varphi_{2}}{k_{01} \cos \varphi_{1}+k_{02} \cos \varphi_{2}}\right\} \text {, } \\
& \text { where } \varphi_{1}=2 \pi T_{M 10} \cdot v, \varphi_{2}=2 \pi T_{M 20} \cdot v \text {. }
\end{aligned}
$$

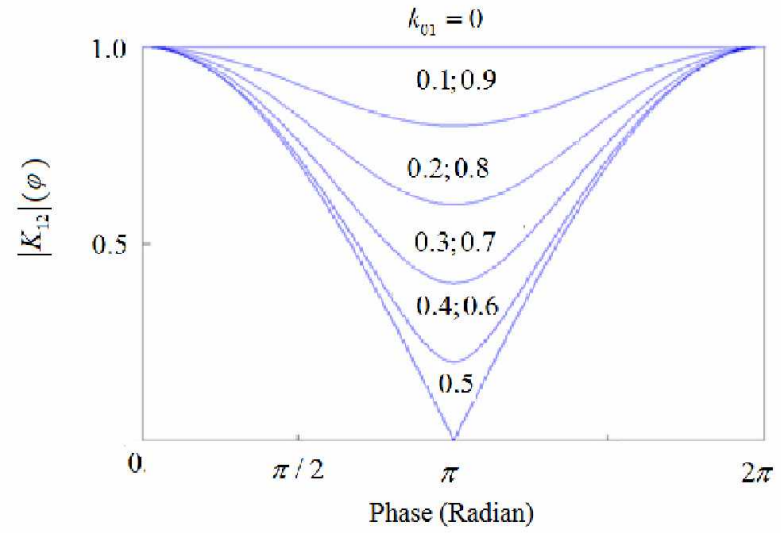

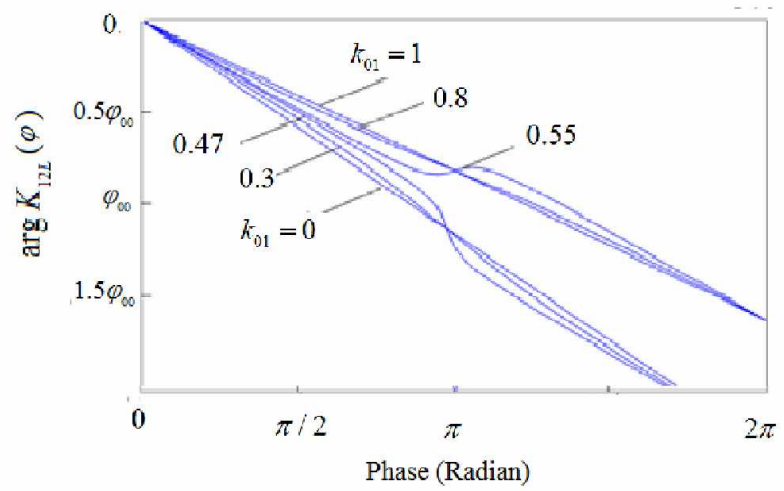

Figure 2. The magnitude and argument plots of the transfer function of the $\mathrm{MZ}$ modulator versus frequency.

Plots of the modulus $\left|K_{12 L}\right|$ and the argument $\arg K_{12 L}(\varphi)$ are presented in Figure 2, where $K_{12 L}=E_{12 L}^{2} / E_{0 L}^{2}$, $\varphi=4 \pi v\left(T_{M 20}-T_{M 10}\right)$. On the y-axis of argument we use $\varphi_{00}=-\pi v\left(T_{M 20}+T_{M 10}\right)$. Noteworthy, the smoothing degree of optical channels according to the excited optical power influences on the modulus and argument of the MZ transfer function.
We attract your attention to the fact that in expression for $\left|E_{12 L}\right|^{2}$ the phase $\varphi_{0 L 1}\left(u_{g}\right)$ is modulated by the harmonic oscillation $\quad u_{g}(t)=U_{1 M Z} \cos \left[2 \pi f_{0} t+\phi_{0 e}+\varphi_{e m}(t)\right] . \quad$ The expression for the function $\left(E_{12 L}\right)^{2}$ versus the oscillation amplitude $U_{0 M}$ and the choice of MZ operation point $\varphi_{0 L 2}$.

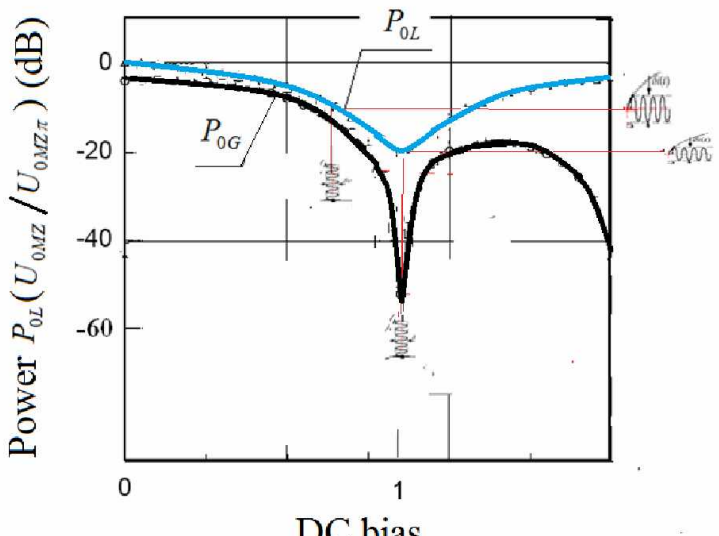

Figure 3. Plots of the power DC bias component of the optical emission $P_{0 L}$ and power AC fundamental component $P_{0 G}$ of $\mathrm{OEO}$ in the PD photo-current (at MZ output)

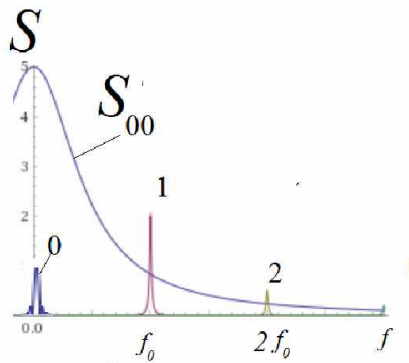

a)

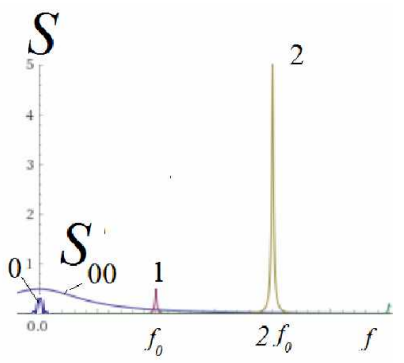

b)
Figure 4. The spectrum structure (qualitative picture) of the PD photo-current of the OEO (Figure 1,c) at $\varphi_{0 M Z}=m \pi / 2$ (a) and $\varphi_{0 M Z}=m \pi(\mathrm{b})$. Designations: DC component -0 , enlarged noise spectrum of DC component - $S_{00}$, fundamental harmonic - 1 , second harmonic - 2

The MZ operation point varies by the DC bias voltage (Figure 3). We introduce the constant half-wave MZ bias voltage $U_{0 M Z \pi}$, which provides the phase difference $\varphi_{0 L 2}$ of 180 degrees between optical oscillations of the first and second MZ channels. Let us suppose for designation convenience that

$$
x=\frac{U_{1 M Z}}{U_{0 M Z \pi}} ; \quad \varphi_{0 M Z}=\frac{\pi U_{0 M Z}}{2 U_{0 M Z \pi}} .
$$

In OEO we use the optical filter, which effectively suppress optical harmonics of carries higher than second $v_{2}=v_{0}-2 f$, and in the OEO circuit with the single-side band 
harmonics from the "left" are suppressed. Then we may present the simplified expression for $E_{12 L}$.

The expression for $\left(E_{12 L}\right)^{2}$ can be rewritten using an expansion on even $J_{2 k}$ and odd $J_{2 k-1}$ Bessel functions. Using the trigonometric formula for cosine of the two angles sum, we keep the first and second harmonics in the record of the carrier expression only from the "left of the carrier". Now we can write expressions without fluctuations as:

$$
\begin{aligned}
& \left(E_{L 12}\right)^{2}=\frac{E_{0 L}^{2}}{2} \gamma\left\{\frac{\left(1+\gamma^{2}\right)}{2 \gamma} \cdot \cos \left(2 \pi v t+\phi_{0 e}\right)\right. \\
& -\cos \left(\varphi_{0 M Z}\right) J_{0}(x) \cdot \cos \left(2 \pi v t+\phi_{0 e}\right) \\
& -\sin \left(\varphi_{0 M Z}\right) J_{1}(x) \cdot \cos \left(2 \pi v t-2 \pi f-\phi_{0 e}\right)-\cos \left(\varphi_{0 M Z}\right) \\
& \left.\times J_{2}(x) \cdot \cos \left(2 \pi v t-2 \cdot 2 \pi f t-2 \cdot \phi_{0 e}\right)\right\} .
\end{aligned}
$$

At spectrum calculation for Bessel functions we use the approximation for $x \in[0 ; 1]$, where $x=U_{1 M Z} / U_{0 M Z \pi}$ :

$$
J_{0}(x) \approx 1-\frac{x^{2}}{4} ; J_{1}(x) \approx \frac{x}{2}-\frac{x^{3}}{16}, \text { and } J_{0}(x) \approx \frac{x^{2}}{8} \text {. }
$$

Now we introduce coefficients $a_{0}, a_{1}, a_{2}$ :

$$
\begin{gathered}
a_{0}=\frac{E_{0 L}^{2}}{2} \gamma\left\{\frac{\left(1+\gamma^{2}\right)}{2 \gamma}-\cos \left(\varphi_{0 M Z}\right) \cdot\left(1-\frac{x^{2}}{4}\right)\right\} ; \\
a_{1}=-\frac{E_{0 L}^{2}}{4} \gamma \sin \left(\varphi_{0 M Z}\right) ; a_{2}=-\frac{E_{0 L}^{2}}{8} \gamma \cos \left(\varphi_{0 M Z}\right) .
\end{gathered}
$$

The PD transfer function (or sensitivity) we define as $S_{P D}$, then taking a noise into account, the expression for PD photocurrent is:

$$
I_{P D}=S_{P D} \cdot\left\langle E_{12 L}(t) E_{12 L \tau}^{*}(t-\Delta t)\right\rangle_{T} .
$$

Having introduced the total phase incursion $\phi_{0 e m Z}$, we obtain following equations for PD photo-current harmonics not taking a noise into account:

$$
\begin{aligned}
& I_{0 P D}=S_{P D} a_{0}-S_{P D}\left(a_{2} / 2\right) x^{2} ; \\
& I_{1 P D}=S_{P D} a_{1}\left(\frac{x}{2}-\frac{x^{3}}{16}\right) \cdot \cos \left(2 \pi f t+\phi_{0 e M Z}\right) ;
\end{aligned}
$$

$I_{2 P D}=S_{P D} a_{2} x^{2} \cos ^{2}\left(\pi f t+\phi_{0 e M Z}\right)$.

Calculations are presented according to the approach accepted in statistic radio engineering. After obtaining of the correlation function, we determine obtain the normalized (by $S_{P D}$ ) spectrum of first and second harmonics and noise contributions as:

$$
\begin{aligned}
& S_{\eta M Z} / S_{P D}=a_{1}^{2} \frac{U_{1 M Z}^{2}}{2} \delta\left(f-f_{0}\right)+a_{2}^{2} \frac{U_{1 M Z}^{4}}{8} \delta\left(f-2 f_{0}\right) \\
& +4\left(a_{1}^{2} \sigma_{L e}^{2}+a_{2}^{2} \sigma_{L e}^{4}\right) \cdot S_{L m}(f)+a_{1}^{2} \frac{U_{1 M Z}^{2}}{2} S_{L \psi 1}\left(f-f_{0}\right) \\
& +a_{2}^{2} \frac{U_{1 M Z}^{4}}{8} S_{L \psi 2}\left(f-2 f_{0}\right)+4 a_{2}^{2} U_{1 M Z}^{2} \sigma_{L e}^{2} \sigma_{L \psi e}^{2} S_{L M \psi}\left(f-f_{0}\right) .
\end{aligned}
$$

In (8) $\delta$ is the delta-function, and two first terms define powers of the first and second harmonics of the photo-current. From these expressions, we can see that levels of first and second harmonics depends on squared coefficients $a_{1}^{2}, a_{2}^{2}$, which in turn are defined by a choice of the MZ operation point and normalized power of optical emission. The third equation in (8) determines the noise spectrum of the constant component, while the fourth one - relatively, the noise spectrum of the fundamental harmonic, while the fifth one the second harmonic.

From this research we can make the following conclusion. The photo-reception, at relatively high optical power arriving on the PD area, is nonlinear and is accompanied by appropriate interaction of the constant intensity component with its alternate component. The level of the constant component of the optical emission of the PD area affects of the noise spectrum of the photo-current of the alternate component of the fundamental photo-current harmonic. To decrease this influence, we can transfer into $\mathrm{MZ}$ operation mode with $180^{\circ}$-difference of phase incursions in MZ optical channels. Figure 3 shows PSD and harmonics, which qualitatively illustrates the formula (8) at different choices of $\varphi_{O M Z}$.

\section{SYMBOLIC EQUATIONS of OEO}

If we introduce into equation system (3) and (11) the operator $p=d / d t$ and take into consideration the total delay time $T_{B C}$ in the open circuit "MZ-C" introducing the FOLD transfer function $K_{B Z}$, we obtain the system of symbolic equations with fluctuating noise sources in the compact form for Laser and OEO:

$$
\left\{\begin{array}{l}
Q_{0}(p) E_{L}=\alpha_{00} E_{L}-\beta_{00} E_{L}\left|E_{L}\right|^{2}+\xi_{S N}, \\
i=0.5\left\langle E_{L} \cdot E_{L}^{*}\right\rangle \gamma \cos \left[\varphi_{0 M Z}+u_{M Z} / U_{0 M Z}\right], \\
{\left[p^{2}+\left(1 / T_{e F}\right) p+\left(2 \pi f_{e F 0}\right)^{2}\right] u_{M Z}} \\
=\left(K_{B Z} / S_{P D}\right) p S_{A}\left(i_{P D}\right) \exp \left(-p T_{B C}\right)+\xi_{U} .
\end{array}\right.
$$

where $K_{B Z}$ is the FOLD transfer function, $T_{e F}$ is the time constant of the electronic filter (EF), $f_{e F 0}$ is the EF natural frequency, $\xi_{U}$ is the Langevinian noise of the electronic amplifier, which is defined by shot noises. In (9), the nonlinear function $S_{A}\left(i_{P D}\right)$ is introduced, which connects the instantaneous current $i_{P D}$ value at the A amplifier input (or the PD photo-current) with the $u_{M Z}$ voltage at the A amplifier output (or $\mathrm{MZ}$ voltage). Equations (9) describe oscillations in the opto-electronic part: closed into a loop of MZ, OF, PD, A, F, C.

Here in (9), we introduce the operator $Q_{0}(p)=Q_{0 F} Q_{0 P}$, where

$$
Q_{0 O F}=\left(p^{2}+\left(1 / T_{0 F}\right) p+\left(2 \pi v_{0 F}\right)^{2}\right) /\left(2 \pi v_{0 F}\right)^{2} ;
$$




$$
Q_{0 P}=\left(p^{2}+\left(1 / T_{2}\right) p+\left(2 \pi v_{12}\right)^{2}\right) /\left(2 \pi v_{12}\right)^{2} .
$$

The total noise component $\xi_{S N}$ is determined as

$$
\xi_{S N}=\left(\xi_{N} \cdot E_{n} \eta_{00}+Q_{P}^{-1} \xi_{P}+\xi_{E}\right) /\left(\left(2 \pi v_{0 F}\right)^{2}\left(2 \pi v_{12}\right)^{2}\right) .
$$

Coefficients $\alpha_{00}$ and $\beta_{00}$ have a sense of amplification and laser saturation, relatively, and are calculated as

$$
\begin{aligned}
& \alpha_{00}=\frac{\left(N_{02}-N_{01}\right) p_{e}^{2} T_{2}}{2 \varepsilon_{0} h} \eta_{00} ; \\
& \beta_{00}=N_{02} T_{1} \cdot G_{0} \frac{p_{e}^{2} T_{2}}{2 \varepsilon_{0} h}\left(\eta_{00}\right)^{2} .
\end{aligned}
$$

The $\eta_{00}$ parameter defines the laser inertia and connects with a life time of $T_{1}$ :

$$
\eta_{00}=\frac{\exp \left(-j 2 \pi v_{0} T_{1}\right)}{\left(1+\left(2 \pi v_{0} T_{1}\right)^{2}\right)^{1 / 2}} .
$$

The first equation of the (9) system has a feature that it is similar by its form to well-studied equations in radio engineering for the double-circuit autonomous oscillator with the arcwise-cubic function of the nonlinear inertial element of the $\mathrm{AC}$ voltage versus current. But coefficients included into equations (9) are expressed via $\mathrm{AC}$ components of the laser physical quantities: a population, a dipole moment, a life-time on the upper operation level, a time constant of the laser optical filter, Langevinian noise sources of optical emission, population and polarization.

A transfer from (9) to differential abbreviated equations allows not only determination the laser power $\left|E_{0 L}\right|^{2}$ in steady-state, but to write abbreviated equations with fluctuations, from which we below obtain expressions for power spectral density (PSD) of the phase and amplitude noises. At that, the laser emission intensity is determined by the expression:

$$
\left|E_{0 L}\right|^{2}=\frac{\alpha_{00}}{\beta_{00}}\left(1-\frac{1}{\alpha_{00} \beta_{00}}\right),
$$

in which the $\left(\alpha_{00} / \beta_{00}\right)$ coefficient is equal to

$$
\left(\alpha_{00} / \beta_{00}\right)=\frac{\left(N_{02}-N_{01}\right)}{N_{02} G_{0} T_{1}} .
$$

Thus, the coefficient ratio of $\left(\alpha_{00} / \beta_{00}\right)$ has got the clear physical sense for lasers. The larger an amplitude of the laser output optical oscillation, the higher a ratio of relative population excess on the level under excitation, and inversely proportional gain factor and lifetime $G_{0} \cdot T_{1}$.

To derive a formula for the noise PSD, we expand the truncated representation $Q_{0}(p)$, denoted as $Q(p)$, into the real and imaginary parts:

$$
Q(p)=Q_{\mathrm{Re}}(p)+j Q_{\mathrm{Im}}(p),
$$

where

$$
Q_{F \mathrm{Re}}=\left(1+F T_{F}\right) \cos \left(F T_{L}\right) /\left(P_{00 L}\left|K_{0 L}\right|\right),
$$

$$
Q_{F \operatorname{Im}}=\left(1+F T_{0 F}\right) \cos \left(F T_{L}\right) /\left(P_{00 L}\left|K_{0 L}\right|\right) .
$$

The $T_{L}$ is delay in the cavity, $\left|K_{0 L}\right|$ is the coefficient of a total loss of optical power in the laser feedback loop. Now we present of the total complex noise component by a sum of real and imaginary parts as:

$$
\xi_{S N}=\xi_{S N \mathrm{Re}}+j \xi_{S N \mathrm{Im}} .
$$

Using the standard approach to abbreviation of differential equations with fluctuating sources, the Fourier transform, the Wiener-Khinchin theorem, we obtain single sideband (SSB) PSD of the laser amplitude and phase noise. The $\xi_{S N R e}$ real part has in spectral representation a view of $S_{S L \mathrm{Re}}$, while the imaginary part $\xi_{S N \mathrm{Im}}$ has a view $S_{S L \mathrm{Im}}$.

SSB PSD equations obtained from (12) for the laser phase noise, which operates in quasi-stationary mode (single-mode and single-frequency) have the following form:

$$
S_{P L}=\frac{\left(Q_{F \mathrm{Re}}-\sigma_{E L}\right)^{2} \cdot S_{S L \mathrm{Im}}+\left(Q_{F I \mathrm{~m}}\right)^{2} S_{S L \mathrm{Re}}}{\left\{\left(Q_{F \mathrm{Re}}-\sigma_{E L}\right) \cdot\left(Q_{F \mathrm{Re}}-S_{0 E}\right)+\left[Q_{F \mathrm{Im}}\right]^{2}\right\}^{2}} ;
$$

where $S_{0 E}=\alpha_{00}-\beta_{00}\left|E_{0 L}\right|^{2}, \sigma_{E L}=\beta_{00}\left|E_{0 L}\right|^{2}$. If for (11) we examine a case when the imaginary part $Q_{F \mathrm{Im}}=0$, $P_{0 L} K_{0 L} \approx 1$, and there is a small delay, i.e., $\cos ^{2}\left(F T_{L}\right) \approx 1$, $\sin ^{2}\left(F T_{L}\right) \approx 0$, then expression (11) for PSD gets the classical form:

$$
S_{P L}=\frac{S_{S L I \mathrm{~m}}}{\left(1+T_{0 F} F-\alpha_{00}+\beta_{00} P_{0 L}\right)^{2}} \approx \frac{S_{S L I \mathrm{~m}}}{P_{0 L}\left(T_{0 F} F\right)^{2}} ;
$$

where $P_{0 L}=E_{0 L}^{2}$ is the optical emission power on the PD lightsensitive area.

The (11) expression defines that the power growth and increase of the laser resonator time constant $T_{0 F}$ (or resonator Q-factor increase) leads to reduction of the laser phase noise.

Expressions (11),(12) for SSB PSD of the laser phase noise do not reflect the important property of the laser oscillating system: a presence of the relaxation resonance on the frequency $v_{00 L}$ at the offset from a carrier $v_{0 L}$, i.e., at $F_{00 L}=2 \pi\left(v_{00 L}-v_{0 L}\right)$. We can take this "resonance peak" into account at linearization of (3) system with account of the population equation. At that, the expression for SSB PSD of the laser phase noise take a form:

$$
S_{P L} / P_{0 L} \approx \frac{S_{S L \text { Im }}}{\left(F T_{0 F}\right)^{2}}+\frac{S_{L E} D_{11}{ }^{2}+S_{L N} D_{22}{ }^{2}}{\left(F^{2}-F_{00 L}{ }^{2}\right)^{2}+\left(F \alpha_{00 l}\right)^{2}}
$$

where $F_{00 L}=\left(1 / T_{1 L}\right)\left(\left(T_{0 F} / T_{1}\right) \alpha_{0}-1\right)^{1 / 2}, \alpha_{0}$ is an excess of DC laser pumping over its threshold value, $\alpha_{00 l}$ is a damping decrement, $D_{11}$ and $D_{22}$ are the constant coefficients, and $S_{L E}, S_{L N}$ are. Relatively, spectral densities of impacts in (3) $\xi_{E}, \xi_{N}$. Figure 5 shows the plot 1 of PSD of the laser phase noise calculated by formula (13) for 
$S_{S L \mathrm{Im}} \approx S_{L E} D_{11}{ }^{2}+S_{L N} D_{22}{ }^{2} \approx-105 \mathrm{~dB} / \mathrm{Hz}, F_{00 L} \approx 14 \mathrm{kHz}$,

$T_{0 F}=10^{-7} \mathrm{~s}$.

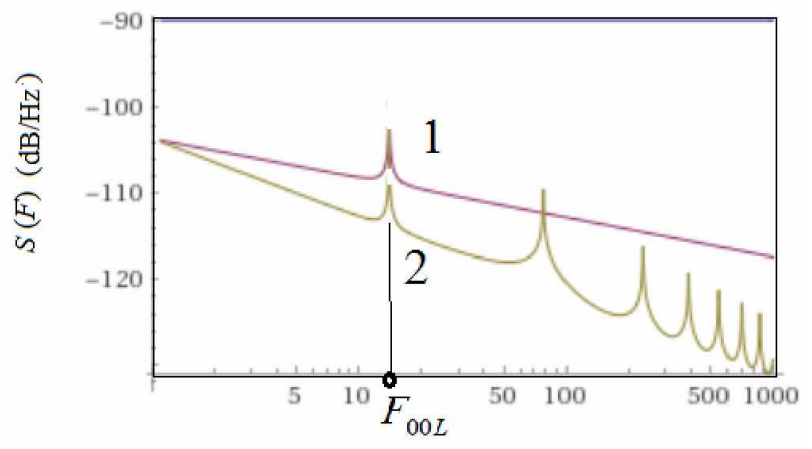

Offset frequency $(\mathrm{kHz})$

Figure 5.Laser phase noise SSB PSD (curve 1), calculated by

(13), and OEO phase noise SSB PSD (curve 2).

\section{OEO PHASE NOISE SSB PSD}

From equations (9) with account of (10) for nonlinear characteristic of the A amplifier (Figure 1) as a cubic polynomial $i_{A}(u)=\alpha_{e 0} u-\beta_{e 0} u^{3} \quad$ (where $u$ is the instantaneous voltage at the amplifier input, and the average slope of this characteristics is $\left.\sigma_{U}=\alpha_{e 00}-(3 / 4) \beta_{e 00} P_{0 G}\right)$ and we can obtain through laser and delay line parameters the power of the opto-Electronic oscillator radiofrequency generation $P_{0 G}$ :

$$
P_{0 G}=\frac{\alpha_{e 00}}{\beta_{e 00}}\left(1-\frac{1}{P_{0 L}\left|K_{B Z}\right| \alpha_{e 00} \beta_{e 00}}\right) .
$$

Similarly to (11) for laser PSD, we obtain from the general symbolic equations (9) the equation for PSD $S_{\Psi}$ of the OEO phase noise. PSD $S_{\Psi}$ reduced to the radiofrequency oscillation power $P_{0 G}$ is determined by expression derived in [12] according to the Evtianov-Kuleshov approach:

$$
S=\frac{S_{\Psi}}{P_{0 G}}=\frac{\left(Y_{a \mathrm{Re}}-\sigma_{U}\right)^{2} \cdot S_{\mathrm{Im} F D N Y}+Y_{a \mathrm{Im}}^{2} \cdot S_{\mathrm{Re} F D N Y}}{U_{0}^{2}\left\{\left[Y_{a \mathrm{Re}}-\sigma_{U}\right] \cdot\left[Y_{a \mathrm{Re}}-1\right]+\left[Y_{a \mathrm{Im}}\right]^{2}\right\}^{2}}
$$

where $S_{\operatorname{Im} F D N Y}=S_{\operatorname{Im} F D N Y}(F), S_{\operatorname{Re} F D N Y}=S_{\operatorname{Re} F D N Y}(F)$ are the inphase and quadrature components of fluctuations determining by the joint noises of a laser, a photodiode, and in the amplifier the OEO closed loop; $F$ is the analysis frequency $\omega$ offset from the generation frequency $\omega_{0}, \quad\left(F=\omega-\omega_{0}\right)$; $Y_{a \mathrm{Re}}=Y_{a \mathrm{Re}}(F), Y_{a \mathrm{Im}}=Y_{a \mathrm{Im}}(F)$ the in-phase and quadrature components of the OEO control conductance (abbreviated representations) $Y_{a}=Y_{a \mathrm{Re}}+j Y_{a \mathrm{Im}}$ :

$$
Y_{a \mathrm{Re}}=y_{M} \frac{\left(1+F T_{F}\right) \cos \left(F T_{B C}\right)}{P_{0 L}\left|K_{B Z}\right|},
$$

$$
Y_{a \mathrm{Im}}=y_{M} \frac{\left(1+F T_{F}\right) \sin \left(F T_{B C}\right)}{P_{0 L}\left|K_{B Z}\right|},
$$

where $\left|K_{B Z}\right|$ the modulus of the transfer function of the open circuit OEO, $T_{B C}$ is the total delay time of oscillations in the OEO open loop including a delay in the OF fiber, $y_{M}$ is the input normalized conductivity of the MZ modulator.

Let us consider the case of large delay and we take into account the laser SSB PSD as in (13). Let in (14) $S_{\operatorname{Im} F D N Y}=S_{\operatorname{Re} F D N Y}=N_{s p} h v$, where the $N_{s p}$ is a number of spontaneous photons received by $\mathrm{PD}$, while $Y_{a \mathrm{Re}}$ and $Y_{a \mathrm{Im}}$ are defined as (18) and (19). Then the function of OEO phase noise PSD (17) can be represented as

$$
S=\frac{S_{\Psi}}{P_{0 G}}=\frac{K_{2} C_{A} h N N_{s p}}{P_{0 G}},
$$

where $C_{A}$ - the constant coefficient, the $K_{2}$ coefficient depends on the delay time in the optical fiber and on the laser optical power and it is equal

$$
K_{2}=\frac{\sigma_{U}^{-2}}{\frac{\left(1+F T_{F}\right)^{2}}{\left(P_{0 L}\left|K_{B Z}\right| \sigma_{U}\right)^{2}}-2 \frac{\left(1+F T_{F}\right) \cos \left(F T_{B C}\right)}{P_{0 L}\left|K_{B Z}\right| \sigma_{U}}+1}
$$

From (17), (18) we see that the laser phase noise are suppressed with OF length growth, increase of the total transfer function in the OEO loop, the laser power as well as $\alpha_{e 00}$ and $\beta_{e 00}$ choice.

Figure 5 shows plots of (13), (18) which are limited functions of OEO of the phase noise PSD with account of small noises of PD and the A amplifier, at laser phase noise for the offset frequency $1 \mathrm{kHz}$ equaled to about $-120 \mathrm{~dB} / \mathrm{Hz}$, at laser power $20 \mathrm{~mW}$, the delay of $T_{B C}=5 \cdot 10^{-6} \mathrm{~s}$ (the OF length is $100 \mathrm{~m}$ ), $\sigma_{U}=1$. We see that the first peak is defined by the laser phase noise PSD, and average suppression of the phase noise for $50 \mathrm{kHz}$ offset is more, that $-10 \mathrm{~dB} / \mathrm{Hz}$.

Figure 6 shows the OEO phase noise PSD versus delay time $T_{B C}$ (or the length of the optical fiber). This calculation is fulfilled on the basis of formulas (17), (18) with about the same assumptions and values of the laser phase noise and main OEO parameters as in Figure 5. It should be noted that at the optical fiber length of $2 \mathrm{~km}$ the uniform suppression of the laser phase noise is achieved in the offset range 1 ...50 kHz. Calculation of the phase noise suppression factor $K_{2}(F)$ suppression factor according to (18) is presented in Figure $7 \sigma_{U}=1$ $\sigma_{U}=1: T_{B C} / T_{F}=1, P_{0 L}\left|K_{B Z}\right|=2$ (curve 1); $T_{B C} / T_{F}=10$, $P_{0 L}\left|K_{B Z}\right|=2$ (curve 2); $T_{B C} / T_{F}=10, P_{0 L}\left|K_{B Z}\right|=4$ ( 3 curve). It can be seen that increase of delay time from $T_{B C} / T_{F}=1$ (curve 1) to $T_{B C} / T_{F}=10$ (curve 2) results in reduction of $K_{2}$ factor more than 10 times in the rated offset frequency $F \cdot T_{F}$ range $0.05 \ldots 0.5$. For example, $P_{0 L}\left|K_{B Z}\right|=2, T_{B C} / T_{F}=10, T_{B C}=10^{-5} \mathrm{~s}, T_{F}=10^{-6} \mathrm{~s}$. It is shown that at OF length, the further reduction of the OEO phase noise is possible using the PLL (phase-locked loop) system. 
Calculation results are well-agreed with experimental dependences of OEO phase noise SSB PSD, which can be found in [14]. Here, we should remind that first publications on research of frequency stability in OEO with the help of FOLD were fulfilled in 1987-1989 at Radio Transmitter Dept. of Moscow Power Engineering Institute (now TKГ MPEI) while the OEO circuit was offered in [15]

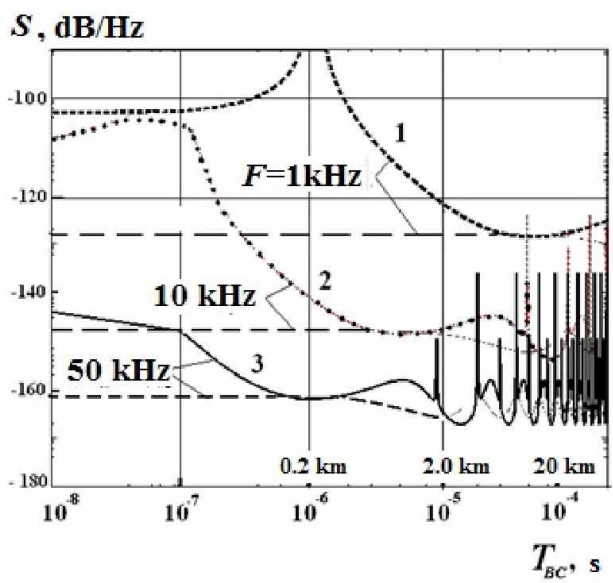

Figure 6. OEO phase noise SSB PSD of MZ modulator depending upon the delay time in $\mathrm{OF}$ at various offsets from the radiofrequency of $10 \mathrm{GHz}$.

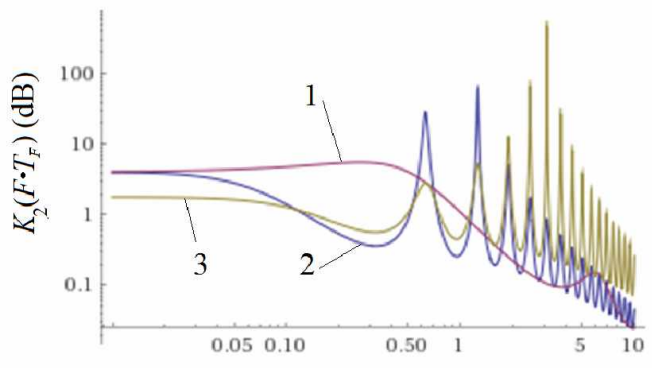

Offset frequency

Figure 7. The phase noise suppression factor $K_{2}$ (equation (18)) versus the rated offset frequency $F \cdot T_{F}$ from the OEO generation frequency $f_{0}$ at different delays in the optical fiber of delay time $T_{B C} / T_{F}$ and the power of the laser optical emission $P_{O L},\left|K_{B Z}\right|$ and $\sigma_{U}=1: T_{B C} / T_{F}=1, P_{0 L}\left|K_{B Z}\right|=2$ (curve 1); $T_{B C} / T_{F}=10, P_{0 L}\left|K_{B Z}\right|=2$ (curve 2); $T_{B C} / T_{F}=10, P_{0 L}\left|K_{B Z}\right|=4$ ( 3 curve $)$.

\section{EXPERIMENTAL INVESTIGATIONS}

Experimental researches were devoted for several experimental OEO of microwave range with various pumping laser diodes, which emit at wavelengths of $1310 \mathrm{~nm}$ or 1550 $\mathrm{nm}$. The maximal output power of optical emission for used laser diodes formed about $10 \ldots 20 \mathrm{~mW}$. Figure 8 shows the photo picture of one piece assembled on the base of the circuit in Figure 1(a). As the photo-detector, we applied the FD on the base of InGaAs. The radiofrequency filter represented the dielectric resonator of microwave range with the loaded Qfactor of 1000 . This resonator was made on ceramics and had a natural frequency $8.2 \mathrm{GHz}$. This breadboard model used the wideband (up to $12 \mathrm{GHz}$ ) modulation of laser emission, which was performed by the Mach-Zender modulator from Hitachi Co. The single-mode light guiders with lengths from $60 \mathrm{~m}$ to $4640 \mathrm{~m}$ were used for experiments. The stable generation of single-frequency oscillation at frequency close to $8.2 \mathrm{GHz}$ was observed in OEO system for various OF lengths.

The delay of OEO signal was performed with the help of additional fiber-optical light guider with the $10 \mathrm{~km}$-length and the additional photo-diode. The phase noise level at usage of different lasers formed the value $-100 \ldots-127 \mathrm{~dB} / \mathrm{Hz}$, for offsets $1 \ldots 10 \mathrm{kHz}$ from the microwave sub-carrier frequency under generation and it depends on the spectral line width of laser emission.

Essential reduction of the phase noise by $15 \mathrm{~dB}$ was observed in OEO using the differential delay line on the base of two optical fibers of different length. These experimental functions are well-agreed with theoretical at account of the stabilization effect at OF lengths more than $2000 \mathrm{~m}$.

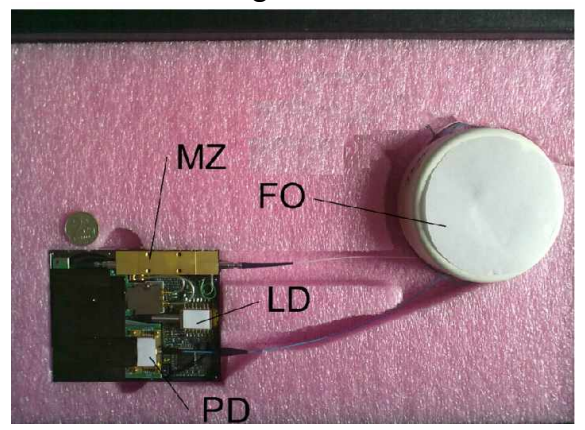

Figure 8. General view of the experimental breadboard of lownoise laser opto-electronic oscillator of microwave range. The mean oscillation frequency is $8 \ldots 10 \mathrm{GHz}$.

\section{CONCLUSION}

Under assumption of the small and large oscillation amplitude at the modulator electrical input, we study OEO as a system in which two oscillation processes are developed on the optical frequency and in radiofrequency. The relatively simple expressions for phase noise PSD of the radiofrequency generation in optoelectronic generator in the mode with the single-side carrier with an account of the laser phase noise. The analysis fulfilled shows that under condition of predominance of laser noises being detected over $\mathrm{v}$ noises of the electronic amplifier and the OEO photo-detector of the filtering system.

For reduction of spurious influence of DC intensity component on the photo-detector we offer to use the modulator operation mode with an offset of the optical channels «pi».

The suppression factor of the OEO laser phase noise at optical fiber lengths from 2 to $10 \mathrm{~km}$ is about $-8 \ldots-10 \mathrm{~dB} / \mathrm{Hz}$ at offset of $F=1 \mathrm{kHz}$. Utilization in OEO of the highlycoherent laser with the phase noise less than $S(F)=-100$ 
$\mathrm{dB} / \mathrm{Hz}$ (at the same offset) is the condition of OEO small phase noises less than $S(F)=-130 \mathrm{~dB} / \mathrm{Hz}$ at the $F=1 \mathrm{kHz}$ offset. The value of the OEO power spectral density is proportional to the spectral line width of the laser optical emission.

\section{Acknowledgements}

Authors express our thanks to $\mathrm{PhD}$ Yu.B. Il'in for manifested interest and participation in discussions.

\section{REFERENCES}

[1.] Aliou Ly, Vincent Auroux, Ramin Khayatzadeh « Highly Spectrally Pure 90-GHz Signal Synthesis Using a Coupled Optoelectronic Oscillator », IEEE Photonics Technology Letters, vol. 30, no.14, pp. 1313-1316, 2018.

[2.] Dan Zhu, Tianhua Du, Shilong Pan «A Coupled Optoelectronic Oscillator with Performance Improved by Enhanced Spatial Hole Burning in an Erbium-doped Fiber », Journal of Lightwave Technology, pp. 3726-3732, (2018).

[3.] Zhuansun Xiaobo and etc, « Low phase noise frequencymultiplied optoelectronic oscillator using a dual-parallel Mach-Zehnder modulator », Optical Engineering 57(08), p. 086101, (2018).

[4.] A. G. Correa-Mena and etc « Performance Evaluation of an Optoelectronic Oscillator Based on a Band-Pass Microwave Photonic Filter Architecture », Radioengineering, 26(3), pp. 642-646, (2017).

[5.] C. X. Li et al., «A Novel Optoelectronic Oscillator with SeriesCoupled Double Recirculating Delay Lines », Advanced Materials Research, Vols. 986-987, pp. 1730-1733, (2014).

[6.] Xihua Zou, Xinkai Liu, et al., « Optoelectronic Oscillators (OEOs) to Sensing, Measurement, and Detection », IEEE Journal of Quantum Electronics 52(1):0601116, (2016).

[8.] X. S. Yao and L. Maleki, « Optoelectronic microwave oscillator », J. Opt. Soc. Amer. B, Opt. Phys., vol. 13, no. 8, pp. 1725-1735, (1996).

[9.] A. A. Savchenkov, A. B. Matsko, V. S. Ilchenko, and L. Maleki, "Optical resonators with ten million finesse," Opt. Express 15, 67686773 (2007).

[10.] J. J. McFerran, E. N. Ivanov, A. Bartels, G. Wilpers, C. W. Oates, S. A. Diddams, and Hollberg, "Low-noise synthesis of microwave signals from an optical source," Electron. Lett. 41, pp. 650-651 (2005).

[11.] Russian patent on invention №2282302 RU, MПK 37 H03 C3/00. « Former (generator) of frequency-modulated signal / Bortsov A. A., Il'in Yu.B. - 10 p. 2004r. (in Russian)

[12.] Zhalud V., Kuleshov V. N. « Noise in semiconductor devices. Under edition of A.K. Naryshkin. - Moscow: Soietskoe Radio, 1977. -416 p. (in Russian)

[13.] Bortsov A. A., Il'in Yu. B. « Laser Spectral Line Effect on RF Phase and Amplitude Noise of an Opto-Electronic Oscillator// Radiotekhnika. No. 2, 2010. - pp. 21-31. (in Russian)

[14.] C.W. Nelson and etc. // Microwave optoelectronic oscillator with optical gain, IEEE, №12, v. 31, pp.152-157, 2007.

[15.] V.V. Grigor'yants, Yu. B. Il'in, « Laser optical fibre heterodyne interferometer with frequency indicating of the phase shift of a light signal in an optical waveguide », Quantum and Quantum Electronics, 21(5), pp. 423-427, (1989).

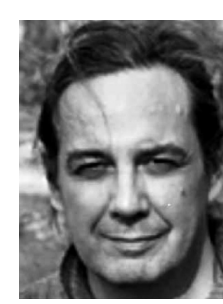

Alexander A. Bortsov, born in 1959, in Moscow, Ph.D. in Engineering, Graduated from MPEI in 1982. PhD thesis was on the theme "Optoelectronic oscillator with the QW laser diode". Author of more 40 scientific publications, among them 30 scientific papers, one book «Quantum Opto-Electronic Oscillator», three USSR copyright certificates on invention, 4 Russian patents, more than 20 technological reports on various conferences, including international. Field of current research: the theory and technique of the opto-electronic oscillator, lasers, QW lasers, phase noise in such oscillators.

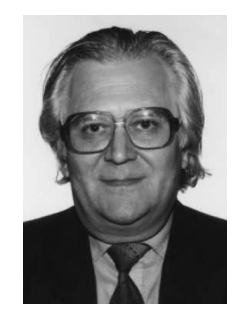

Sergey M. Smolskiy, born in 1946, Ph.D. in Engineering, Dr.Sc. in Engineering, full professor of Department of Radio Signals Formation and Processing of the National Research University "MPEI". He was engaged in theoretical and practical problems concerning the development of modern transmitting cascades including the short-range radar. In 1993 he defended the Doctor of Science thesis and now he works as a professor of Radio Signals Formation and Processing Dept. Academic experience - over forty years. The list of scientific works and inventions contains over three hundreds of scientific papers, 15 books, more than 100 technological reports on various conferences, including international. The active member of International Academy of Informatization, International Academy of Electrotechnical Sciences, International Academy of Sciences of Higher Educational Institutions. The active member of IEEE. The scientific work for the latter fifteen years is connected with conversion directions of short-range radar systems, radio measuring systems for fuel and energy complex, radio monitoring system etc. 